\title{
From in vivo to in vitro models to assess bioavailability properties of Plutonium compounds
}

\author{
Olivier Grémy ${ }^{1}$, Nina Griffiths ${ }^{1}$, Laurent Miccoli ${ }^{1}$, and Anne Van der Meeren ${ }^{*}, 1$ \\ ${ }^{1}$ Laboratoire de Radiotoxicologie, CEA, Paris-Saclay University, 91297 Arpajon - FRANCE
}

The understanding of the biodistribution of actinides following internal contamination is an important step in the process of dose estimation and risk assessment, as well as in determining an appropriate decorporation treatment. Dose calculation is based, in part, on transferability properties of radiocontaminant. For this purpose ICRP classifies materials according to their absorption from lungs into blood in three types: Fast (F), Moderate (M), and Slow (S). Plutonium $(\mathrm{Pu})$ can exist in multiple forms with differing physico-chemical properties in the work place. It is thus beneficial to develop robust in vitro models as a first approach to predict $\mathrm{Pu}$ compound behavior in vivo and to facilitate dose calculation and decisions for appropriate therapy.

For an estimation of $\mathrm{Pu}$ biodistribution following inhalation, pulmonary administration in rats is the most common approach. However, animal models remain time consuming and are not appropriate for the study of a great number of compounds. In addition, an a priori knowledge for the categories of a Pu compound involved in a contamination incident would be of great help for an early dose assessment. Therefore, we have developed in vitro models to i) mimic uptake and release by macrophages [1] and ii) determine bioavailability properties of radiocontaminants to predict the in vivo behavior [2].

In the present study, using in vivo and in vitro models, we evaluated the behavior of a $\mathrm{Pu}$ compound of unknown physico-chemical properties. Pu solution with unknown properties was intra-tracheally administered in rats, or used for in vitro contamination of macrophages as well as in the acellular model for bioavailability prediction (fig 1). Data were then compared to our historic data obtained using three forms of $\mathrm{Pu}$ (colloid, nitrate, citrate) of distinct solubility properties [3] and that we chose to represent the three ICRP transportability classes (respectively S, M and F).

Two weeks following pulmonary contamination in rats, retention in lungs, bone and liver was evaluated by alpha activity measurement using liquid scintillation counting. Bronchoalveolar lavages (BAL) were carried out to recover alveolar macrophages and quantification of the retained Pu activity. Both the human macrophage-like THP-1 cells and alveolar macrophages from naïve non-exposed rats were exposed to the unknown $\mathrm{Pu}$

* Corresponding author: anne.vandermeeren@cea.fr 
compound in solution for two hours to assess $\mathrm{Pu}$ uptake. Thereafter, Pu-loaded macrophages were cultured in a transwell ${ }^{\circledR}$ culture system and $\mathrm{Pu}$ release was measured over 7 days. Finally, in a third experimental approach, agarose gels were prepared so $\mathrm{Pu}$ compound solution was entrapped in the static phase representing the retention compartment, and the released fraction in the dynamic phase was an indication of the intrinsic solubility properties of the contaminant.

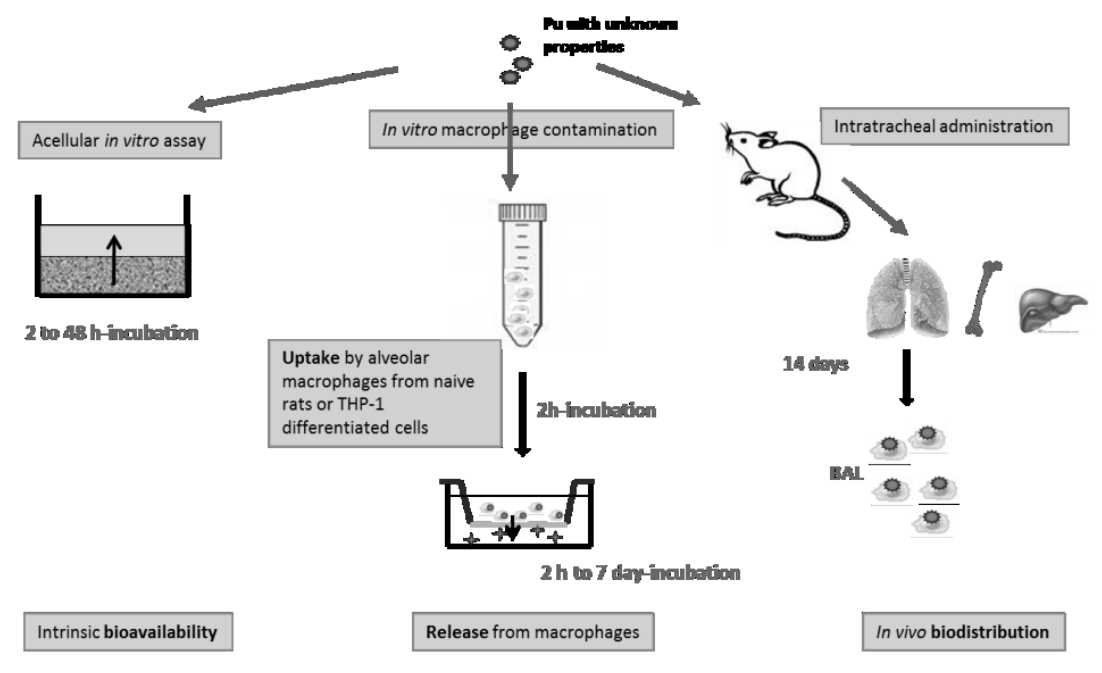

Fig.1. Design of the experimental approach

By comparing the data to previous results obtained in the laboratory using $\mathrm{Pu}$ as colloid, nitrate or citrate form, and whatever the model used, we demonstrated that the unknown $\mathrm{Pu}$ compound behaved very similarly to Nitrate. Therefore, were able to classify the $\mathrm{Pu}$ compound as a type $\mathrm{M}$ default absorption type, according to ICRP classification. These results suggest that the in vitro methods could be used as a first approach to predict the biodistribution of a contaminant, and help during the dose assessment process to classify the contaminant according to its solubility properties. In addition, the development and use of in vitro models to predict retention and propose transportability classes fulfill the aims of the 3 Rs (Reduce, Refine, Replace) with regard to animal experimentation.

Results presented here were obtained in the frame of the AREVA NC-CEA collaboration.

[1] A. Van der Meeren, A. Moureau, D. Laurent, P. Laroche, JF. Angulo-Mora. Toxicol. in Vitro 37, 25 (2016)

[2] CEA-AREVA NC International Patent Application WO2017109024 published June 29, 2017.

[3] A. Van der Meeren, O. Grémy, D. Renault, A. Miroux, S. Bruel, N. Griffiths, F. Tourdes. J. Radiat. Res. 53, 184 (2012) 